\title{
CURRENT SCENARIO OF TELEDENTISTRY IN PUBLIC HEALTHCARE IN INDIA
}

\author{
OP Kharbanda MDS, MOrth, M MEd ${ }^{1}$, Harsh Priya MDS ${ }^{1}$, Rajiv Balachandran MDS ${ }^{1}$, Charu Khurana \\ MDS $^{2}$
}

${ }^{1}$ Centre for Dental Education and Research, All India Institute of Medical Sciences, New Delhi, India

${ }^{2}$ National Oral Health Programme, Centre for Dental Education and Research, All India Institute of Medical Sciences, New Delhi, India

\begin{abstract}
India is the largest democracy and the second most populated nation in the world. Although with 190,000 dentists, India ranks top in the absolute number of dental graduates, rural Indians and urban slums remain deprived of quality dental healthcare due to unequal distribution and access. About 1,000 telemedicine nodes have been established by Government/Private/Trust agencies to reinforce the national healthcare delivery system in India however an organised and dedicated teledentistry network is non-existent but for the Collaborative Digital Diagnosis System (CollabDDS). CollabDDS was developed in India for tele-consultation, diagnosis, remote education and as a data repository. It is a remote expert dental programme served between three dental schools with the Centre for Dental Education and Research at the All India Institute of Medical Sciences in New Delhi. There are some major challenges which exist and need to be addressed including lack of government initiatives, reimbursement schemes, data protection laws, technical infrastructure, advanced biological sensors, bandwidth support, orientation among doctors, and linguistic diversity, along with patients' fear and unfamiliarity. With an area of 3,287 million square kilometres, an urban-rural divide, inaccessible areas, the country is an ideal setting for the provision of eHealth. This paper highlights the present status, challenges and future of teledentistry in India.

Keywords: telemedicine; teledentistry; eHealth; CollabDDS; India

Kharbanda O et al.. JISfTeH 2019; 7:e10(1-8).

DOI: https://doi.org/10.29086/JISfTeH.7.e10

Copyright: ${ }^{\circledR}$ The Authors 2019

Open access, published under Creative Commons Attribution 4.0 BY International Licence
\end{abstract}

\section{Introduction}

India is the largest democracy and the second most populous country in the world after China. It is a mixed economy of 1.3 billion people who make up $17.5 \%$ of the world's total population. The majority of Indians $(69 \%)$ live in rural areas. ${ }^{1}$ India is made up of 29 states, six union territories and one national capital region. The public health facilities in the state are supported and managed by their respective state health administration. The federal government determines policies and supports national healthcare programmes of significance and priority in the country. ${ }^{2}$ Over the last five decades elimination of malnutrition, control of infectious diseases and the improvement of infant mortality rate has received significant attention in healthcare. The average life expectancy in India which was 31 years in 1947 just after independence from British Raj, has now improved to 68.8 years. India ranks $125^{\text {th }}$ in the world life expectancy ranking. ${ }^{3}$ In contrast, dental and oral health received little attention. In recent years the focus on healthcare, and policies and funding has shifted to non-communicable diseases including oral and dental health, cancer and quality of life.

The burden of dental diseases and their consequent sequelae is enormous in India. ${ }^{4}$ To reduce sufferings and morbidity associated with oral health diseases, the Government of India launched the first National Oral Healthcare Programme in collaboration with Centre for Dental Education and Research at the All India Institute of Medical Sciences, New Delhi in 1999. The National Oral Health Programme which was initiated as a pilot programme was first implemented in one district each in five states across the country. The strategy for this programme has been oral health education with extensive use of information, education and communication materials to generate awareness on dental health in the public. ${ }^{5}$

In the $12^{\text {th }}$ Five Year Plan of the government of India, in 2012, an allocation was made by the Ministry of Health Family Welfare for the formulation and implementation of a Basic Package on Oral Health. The package included human resource support, infrastructure development, capacity building and monitoring of dental public health as well as research through the setup of national, state and district Oral 
Health Cells. ${ }^{6}$ The prevention of dental diseases was the primary focus, with little concern for the delivery of dental care. Ayushman Bharat is the recent and a novel healthcare initiative of the federal government which includes a limited component of dental care. Under this scheme, poor people in India become eligible for cashless treatment worth INR 5 lakh ( USD 7,100) in all public funded and select private hospitals across India. Moreover, 150,000 new health and wellness centres will start and provide free health treatment and low-cost medicines for poor and needy people. ${ }^{7}$

Currently, the 313 Dental Colleges in India graduate approximately 27,000 dentists (BDS) every year. With this, there has been an improvement in the dentist to population ratio, which is currently $1: 10,271$. The urban and affluent locations attract more dentists and specialists compared to rural and low-income locations. ${ }^{8}{ }^{9}$ Rural Indians have to face diverse challenges including geographic remoteness, lack of public transportation and poverty to access oral healthcare resulting in pain, suffering and mortality. ${ }^{10}$ The use of India's advances in the field of information and communication technology (ICT) has also influenced healthcare delivery. The National Knowledge Network $(\mathrm{NKN})^{11}$ is a fast and robust Indian network which is capable of providing secure and reliable connectivity. In India, NKN with its multigigabit capability aims to connect all universities, research institutions, libraries, laboratories, healthcare and agricultural institutions across the country to address the collaboration between research and innovation.

\section{Telemedicine/Teledentistry in India}

The Department of Information Technology and the Ministry of Communications and Information Technology launched a pilot telemedicine project through the Indian Space Research Organisation (ISRO) in 1999. Around 1,000 telemedicine nodes were established across the country by Government/Private/ Charitable Trust agencies. ISRO contributed a total of 414 nodes of which 384 were located at remote medical treatment locations that were linked to 60 speciality hospitals. The Apollo Telemedicine Networking Foundation has also established 500 active centres including 164 electronic urban primary health centre and 115 teleophthalmology centres. It has about 150 franchises all over the country. The main objectives were to reinforce the national healthcare delivery system. ${ }^{12-14}$

In 2001, ISRO pilot project progressed to a national telemedicine resource centre at Sanjay Gandhi Post Graduate Institute of Medical Sciences in Lucknow. The national centre is supported by five Regional resource centres across India. Besides telemedicine consults, the national telemedicine resource centre has organised health camps in rural places and provided healthcare access through mobile telemedicine units in mass assemblage at 'Maha-Kumbh Mela' (Allahabad) in 2001, 'Puri-RathYatra' (Odisha) in 2009..$^{15}$

Current telemedicine practices in India

India implemented the Pan African e-Network Project (2009-
2017) for global healthcare. ${ }^{16}$ This project created significant linkages for tele-education, telemedicine, Internet, video conferencing and voice over Internet protocol (VoIP) services. The Pan African e-Network made available the facilities and expertise of some of the best universities and super-speciality hospitals in India to 48 countries of Africa. Some countries opted not to join the network and several no longer participate in the programme. At a total cost of INR 542.90 crores (USD 78 million), the Ministry of External Affairs has set up, patient end locations in 12 Indian Super Specialty Hospitals and 460 telemedicine consultations and around 2,439 continuing medical education (CME) sessions in English and 167 CME sessions in the French have been conducted on medical and dental subjects in the last three years. ${ }^{16}$

Telemedicine in healthcare delivery: initiatives through AIIMS, New Delhi

The All India Institute of Medical Sciences (AIIMS) has been a regional resource centre for the 'National Medical College Network' since 2014. AIIMS is expected to arrange telehealth facility including teleconsultation, telediagnosis, tele-education and telereferral to nine medical colleges and hospitals across several states (Delhi, Uttarakhand, Madhya Pradesh, Rajasthan and Chhattisgarh) in the north-central region in India.

\section{Tele-CME Programme}

AIIMS conducts case discussions between various departments (Gastrointestinal Surgery, Paediatric Pulmonology, Paediatric Rheumatology and Orthopaedics) at AIIMS and one medical college each in above mentioned nine states. The case presentations are being conducted on a weekly basis for the last two years. It popularity supports its usefulness and enhanced clinical benefits for the participants. Tele-Evidence Programme

The role of physicians often extends beyond the provision of direct patient care and includes possible appearance in court as a professional or expert witness to give their testimony in various legal cases. Court visits can be minimised through the provision of tele-evidence to testify with the obvious benefit of saving the precious clinical time of the doctors and resources. ${ }^{17}$ Tele-evidence services are provided at the Postgraduate Institute of Medical Education and Research at Chandigarh and AIIMS New Delhi.

\section{Telemedicine/ teledentistry networks in the States}

The documented information on teledentistry per-se is not available from any of the 29 states, six union territories and one national capital region. Three states are specifically working in the field of teleconsultation, of which Maharashtra has implemented tele-consultation for dental related problems as well. Maharashtra state in the west of India extended telemedicine facilities to 23 district hospitals and 39 sub-district/rural hospitals in 2014-15. These nodes were connected to the six speciality hospitals. The Sir J.J. Hospital located in the state capital of Mumbai serves as the control hub. This tele-consultation facility has provided 
expert opinion to 198,836 patients including 3,767 patients with dental problems. The facility is also utilised for advanced education to doctors and para-medical staff at district/sub-district / rural hospital on 679 topics. ${ }^{18}$

In the state of Odisha, "Odi-telecon" has had 16,556 teleconsultations and telefollow-ups since its introduction in 2001. A total of 490 training courses were held through this network to benefit of medical professionals and para-medical staff from August 2001 to May 2017. Sixteen batches of postgraduate trainees in the three Government medical colleges of Odisha have benefitted through online teaching on 3,141 topics. $^{19}$

In Kerala state, The 'Tele-Oncology Network' was the first to provide telemedicine services for early detection, follow-up and pain relief for cancer patients from rural areas. 'ONCONET-Kerala' telemedicine project was completed by the Centre for Development of Advanced Computing (CDAC) and the Regional Cancer Centre in Trivandrum. This project, launched in 2001 also included the creation of a web-enabled Hospital information system 'TEJHAS' (Telemedicine enabled Java-based Hospital Automation System), an electronic database of patient's medical records, easily accessible to all medical centres in the region. ${ }^{20}$

The Telemedicine Society of India was formed during the National Conference on Telemedicine held in Lucknow in April 2001. The participants resolved to form a scientific society dedicated to Telemedicine and the society hosts an annual conference. An International Conference on Telemedicine (INTELMED 2005) was hosted by the Indian Space Research Organisation (ISRO) at Bangalore. ${ }^{21}$ Although a significant number of articles have appeared in Indian journals and magazines about teledentistry in India, these have been limited to creating awareness. There is no formal organisation in the field of teledentistry in India.

ECHO India- $A$ revolution in medical care and education Project ECHO (Extension for Community Healthcare Outcomes) is a collaborative model of medical education and care management that empowers clinicians everywhere to provide better care to more people, right where they live. ECHO India was started as a not-for-profit Trust in 2008. Currently, teleECHO clinics in India are run by 15 hubs to tackle different states. It is a concept of weekly or fortnightly virtual clinics that use teleconferencing, combined with mentoring and patient case presentations to bridge the gap in healthcare resources by using best specialists to reach out to underserved areas.

National Institute of Cancer Prevention and Research (NICPR) being a partner with project ECHO, conducts 14 weeks virtual advanced cancer screening training programme for dentists and community health specialists interested in oral cancer, screening. The purpose of the training programme is to empower screening for oral potentially malignant lesions and oral cancer and learn to promote counselling the community in hazards of tobacco and tobacco cessation. $^{22}$

\section{CollabDDS: Collaborative Digital Diagnosis System}

The current practice of teledentistry to accomplish teleconsultation, diagnosis and education in India started with the implementation of CollabDDS. ${ }^{23}$ CollabDDS is unique software developed to address the logistic issues in quality healthcare delivery and education in rural/remote areas. The digital system provides an integrated online environment to visualise medical and dental images for diagnosis and treatment planning. CollabDDS is a successful outcome of the synergy of specialists from varied disciplines and knowledge domains.

This system was developed in collaboration by:

- CollabCAD Development Group, National Informatics Centre, New Delhi

- Department of Orthodontics and Dentofacial Deformities, Centre for Dental Education and Research, AIIMS, New Delhi

- Department of Radiodiagnosis, AIIMS- New Delhi

- OrthoCAD Lab, Mechanical Engineering Dept., IIT Bombay, Mumbai

- CSIO, Central Scientific Instruments OrganizationChandigarh

The first version of the CollabDDS v1.0 was released in July, 2012 and has undergone upgrade and development based on feedback from experts and the latest version 2.4 was released in August, 2018. CollabDDS allows real-time collaboration at two or more remote locations using the high bandwidth and low latency of national knowledge network. (Figure1) The initial pilot testing was done between AIIMS, New Delhi, Government Dental College and Hospital, Ahmedabad, Government Dental College, Goa and King George's Medical University, Lucknow.

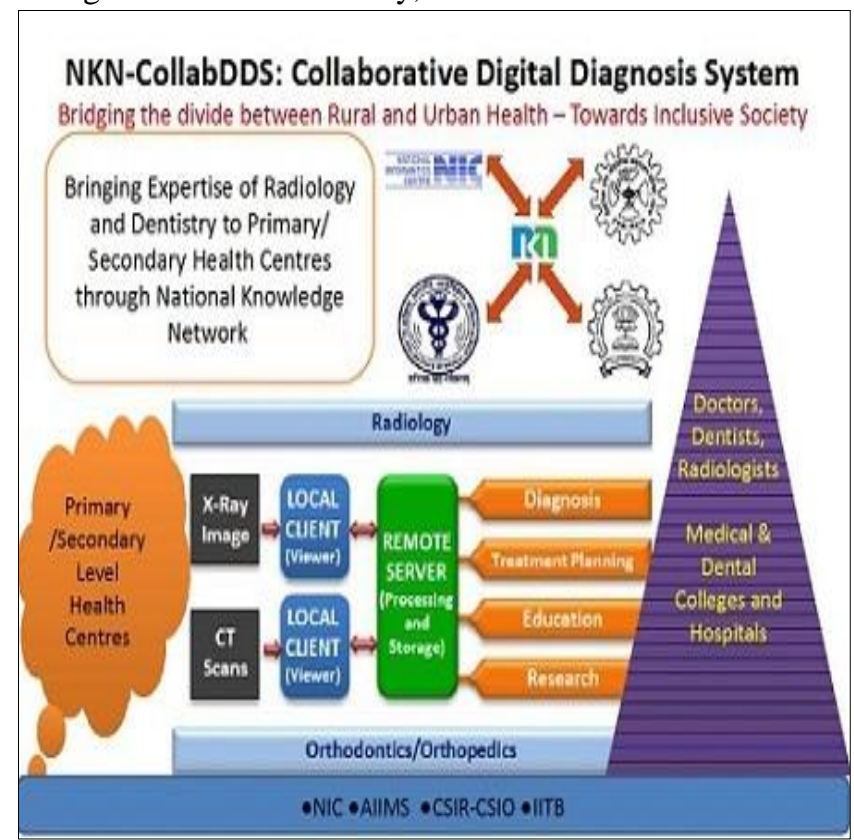

Figure 1. Architecture of Collaborative Digital Diagnosis System (CollabDDS). 
The advantages of CollabDDS are:

- Efficient digital workflow of x-rays/ CT Scans/ MRI images

- Improved diagnosis and treatment through collaborative consultation

- Enhancement of quality of medical services in rural areas

- Reliability, security and accessibility of medical records

- Education based on real-time expert diagnosis

The pilot implementation of CollabDDS at Medical and

Dental Colleges was initiated as part of the NKN Model project. The colleges selected for the pilot implementation are given in Table 1.

Table 1. Medical and Dental colleges selected for the pilot implementation of CollabDDS.

\begin{tabular}{|l|c|}
\hline Medical Colleges & Cases covered \\
\hline SN Medical College, Agra & 34 \\
\hline $\begin{array}{l}\text { Andhra Medical College and King George } \\
\text { Hospital, Visakhapatnam }\end{array}$ & 59 \\
\hline Sawai Man Singh Medical College, Jaipur & 88 \\
\hline $\begin{array}{l}\text { King Georges Medical University, } \\
\text { Lucknow }\end{array}$ & 39 \\
\hline Total Cases covered (Medical) & $\mathbf{2 2 0}$ \\
\hline Dental Colleges Cases covered \\
\hline Government Dental College, Goa & 15 \\
\hline $\begin{array}{l}\text { Government Dental College and Hospital, } \\
\text { Ahmedabad }\end{array}$ & 37 \\
\hline $\begin{array}{l}\text { Faculty of Dental Sciences, King } \\
\text { George's Medical University, Lucknow }\end{array}$ & 1 \\
\hline Total cases (Dental) & $\mathbf{5 3}$ \\
\hline
\end{tabular}

\section{Goals and objectives of the project}

The project has two goals. The first is to provide real-time radiological diagnosis for patients at remote places by con- necting Primary Health Centres with expert radiologists, doctors and dentists at medical and dental colleges and hospitals using suitable ICT tools. The applications are to be rolled out to selected dental and medical colleges to ascertain the usefulness of the applications with the ultimate goal of providing quality oral healthcare to all the citizens of India. The second objective is to use this platform to build a repository of rare clinical cases giving medical and dental students the opportunity to study cases which are currently limited to the centres of excellence.

Pilot implementation of CollabDDS at Dental Colleges ${ }^{23}$ (Case study)

Real-time collaborative sessions between CDER, AIIMS and selected dental colleges were undertaken on a project basis. During the last 18 months, a total of 32 sessions and 53 cases were discussed with remotely placed dental institutions. (Table 2)

Table 2. The total number of sessions and cases discussed with each dental college.

\begin{tabular}{|l|c|c|}
\hline Dental College & Sessions & Cases \\
\hline $\begin{array}{l}\text { Government Dental College and } \\
\text { Hospital, Ahmedabad }\end{array}$ & 19 & 37 \\
\hline Government Dental College, Goa & 12 & 15 \\
\hline $\begin{array}{l}\text { Faculty of Dental Sciences, King } \\
\text { George's Medical University, Lucknow }\end{array}$ & 1 & 1 \\
\hline Total & 32 & 53 \\
\hline
\end{tabular}

Feedback of Teleconsult on CollabDDS

A web-based feedback application was developed to enhance the capabilities and improve the comfort and use of CollabDDS. It includes report generation specific to institute, department, domain, modality, date and case ID. The data could be saved as a Pdf or Excel format. (Figure 2)

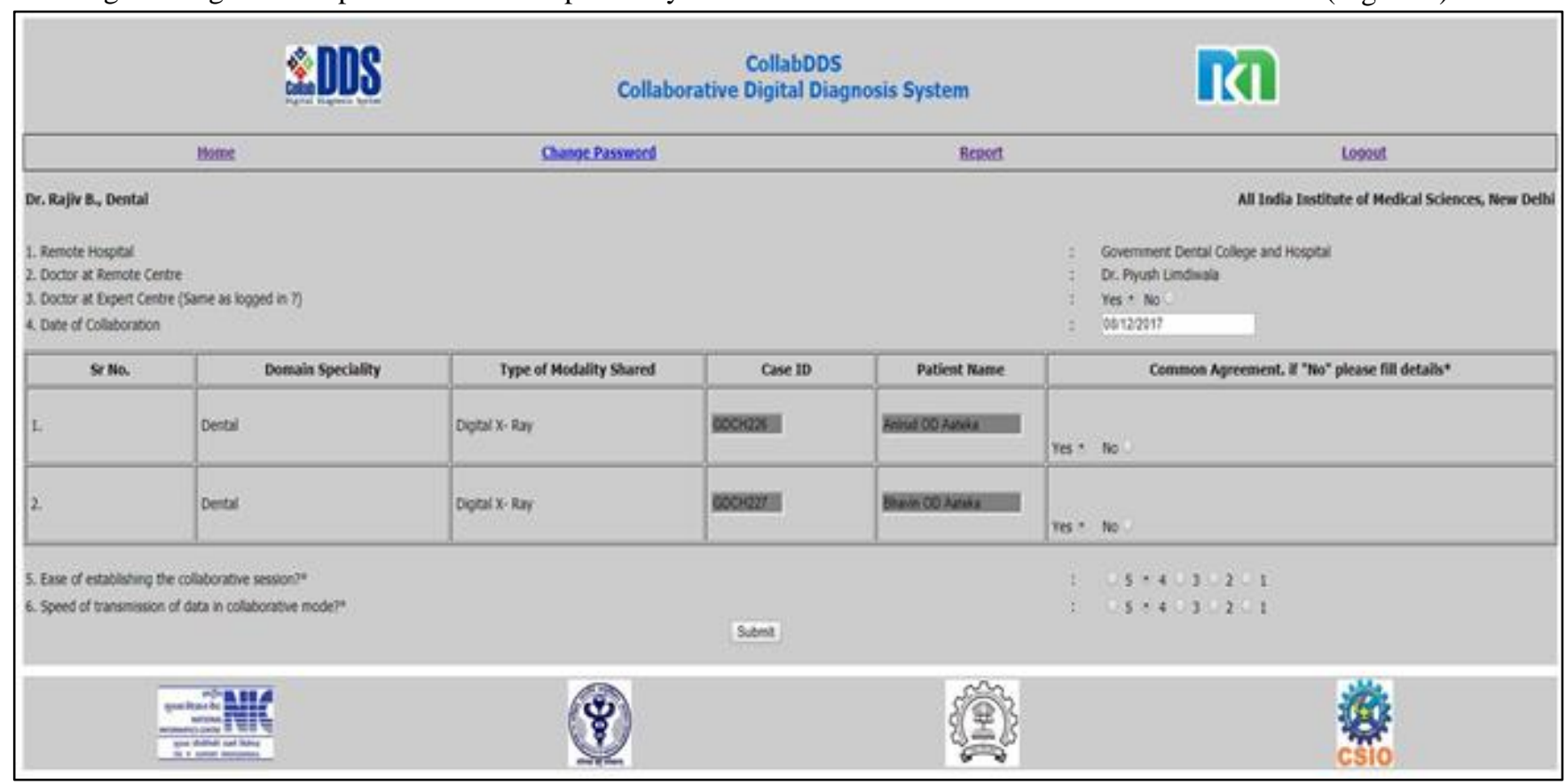

Figure 2. Collaborative Digital Diagnosis System (CollabDDS) report. 
The parameters on which evaluation of diagnosis was made from both sides, remote as well as the expert institution were as follows:

- Ease in establishing the collaborative session

- The speed of transmission of data in the collaborative mode

- To what extent this collaboration helped the remote institution in their diagnosis

- Was there any collective agreement between the remote and expert institution regarding the diagnosis of this case?

The rating was based on the 5 point scale, where 5 represented very good and 1 represented very poor. (Figure 3)

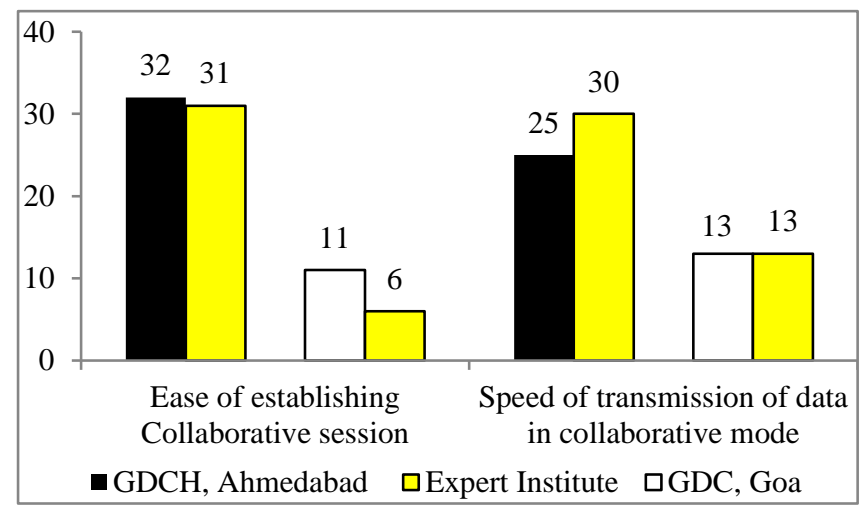

Figure 3. Number of cases with ratings $(>3)$ in in collaborative discussion betweenGovernment Dental College and Hospital (GDCH), Ahmedabad and Government Dental College (GDC), Goa and AIIMS.

Feedback from the participants showed that $75 \%$ cases had a rating of more than 3 and found the collaboration helpful in their diagnosis. Nearly all the cases (96.1\%) showed agreement on diagnosis and treatment planning between remote and expert by modality, domain and department.

\section{'eDantSeva' - A start of telemonitoring in dentistry}

eDantSeva is a web based interactive site and mobile app created for the National Oral Health Programme, by the Centre for Dental Education and Research supported by Ministry of Health, Government of India. 'eDantSeva' has many distinct features, including 'Find a Dental Facility' which locates and maps dental facilities around the geographical location. All 313 dental schools and 1,000 hospitals including primary and community health centres across India have been mapped and can be tracked. ${ }^{24}$

\section{Legal issues}

Ethical and legal issues are major constraint in the progress of telemedicine and teledentistry and this is no different in India. The issues are related to privacy and confidentiality, security of the data, safety and standards. ${ }^{25}$ The confidentiality of the data transmission through CollabDDS in India is maintained with utmost security. Informed consent of the patient and of referral and consulting doctors are integral to the system. ${ }^{23}$ The Dental Council of India regulates dental practice and education in India. Every practicing dentist must be licenced through registration of his or her qualifications with the Dental Council in the state or region of his practice. Doctors and dentists are advised to obtain written informed consent for telemedicine activities. ${ }^{25}$ There is no formal regulation or licencing required for practising teledentistry in India.

In India, to maintain safety standards of the equipment used in the telemedicine or teledentistry projects, the focus is mainly on using high definition media interface for the sessions. Lack of proven assessment frameworks and standardised technology platforms, particularly for diagnostic devices, are a major reason for the slow adoption of telehealth solutions in India. ${ }^{26,27}$

\section{Data protection law}

India presently does not have any specific legislation governing data protection or privacy for telemedicine or teledentistry. The relevant law dealing with data protection are part of Information Technology (IT) Act, 2000, IT Amendment Act, 2008 and the Indian Contract Act, 1872. Health information is not integrated with technology as in many other countries. However, It is not legal to prescribe any medication through any smartphone applications in India. ${ }^{25,28}$

\section{Financial arrangements}

Although teledentistry looks promising for dental consultation and dental education, its strengths and limitations need to be understood. Teledentistry requires a reliable backup of the communication system and technical support. Network technicians, computer hardware and software technicians and security experts are integral to the team. The communication bandwidth costs provided by private agencies are expensive for most of the hospitals. ${ }^{29}$

Reimbursement for Teledentistry consultation needs to be addressed. The Pan African e-Network project funded by Ministry of External Affairs of India provided remuneration to doctors providing tele-education and teleconsultation of around INR 4,500 ( USD 64) and INR 1,350 ( USD 19) respectively per case after $10 \%$ tax deduction. ${ }^{16}$ However, currently in India, no insurance company has a particular reimbursement scheme for the cost of telecommunication equipment and the remuneration of the consultants who provide the teleconsultation services. ${ }^{25,30}$ Incentivising all the stakeholders involved is a major challenge; without reimbursement from insurance companies, the financial support for these projects is limited to research grants and other limited resources, sustaining such high-maintenance projects after the grant period ends can be a severe problem. ${ }^{30}$

\section{Successes and Failures}

The deployment of the Internet and broadband high-speed connection remain the cornerstones of modern telemedicine and teledentistry and help to make it feasible and successful across remote locations.

In India, currently major networks in operation are as follows:

- National Knowledge Network by National Informatics Centre (NIC) 
- Integrated Services Digital Network (ISDN) by the largest public telecommunication services provider (MTNL)

- Very Small Aperture Terminal (V-SAT) by Indian Space Research Organisation (ISRO)

- State Wide Area Network (SWAN)

- National Optic Fibre Network (NOFN)

NKN provides a fast network through 10 Internet protocol (IP) addresses to all connected institutes at the same time. It is one of the successful systems which helps in data transmission with a high speed of 100 Mbps followed by ISDN (1-10Mbps) and V-SAT (128-756 Kbps) and provides a connection from expert institute to 3 to 50 remote places around. ${ }^{14}$ Other private Internet service providers are Airtel, Jio, Vodafone, Idea Limited, Tata teleservices and Atria convergence technology (ACT). The data on the use of these service providers for teleconsultation is not available.

TeleECHO sessions are popular for medical education, training and support to doctors, paramedical staff and primary healthcare workers. ${ }^{22}$

Technical infrastructure, training and cost of equipment are major constraints in setting up of telehealth projects in India. Logistic issues are always inherent in hardware, software and network connections. Electric power interruption is not uncommon which leads to abrupt disruption of sessions between hub and remote site. 'Packet loss, with poor image quality is a commonly encountered limitation that occurs when any one of the receivers does not have a high-speed connection or has low bandwidth. ${ }^{31}$ The other challenge to the use of teledentistry is lack of awareness. A study by Nagarajappa et al. on knowledge and attitudes towards teledentistry among dentists in Udaipur, Rajasthan reported unbalanced knowledge of teledentistry suggesting the need for awareness programmes to fill the knowledge gaps and instil positive attitudes. ${ }^{32}$

Literacy rate and linguistic diversity is also an important factor which needs to be considered. Only $72.1 \%$ of India's population is literate with over 22 officially recognised languages and over 1,600 mother tongues, which could be a barrier for a patient in one region talking to a doctor in another region. ${ }^{33}$

Most telehealth organisations in India are operating in silos, with very little or no opportunity for cross-learning and knowledge exchange resulting in the slow growth of eMedicine in India. ${ }^{29}$

\section{Future of teledentistry in India}

With an area of 3,287 million $\mathrm{km}^{2}$, an urban-rural divide, inaccessible hilly regions, islands and many tribal areas, India is an ideal setting for telemedicine and teledentistry assisted healthcare delivery. Teledentistry can embrace a whole new treatment opportunity for, dentists, specialists and dental hygienists to work together. The dental colleges can act as a "hub" for providing teledentistry consultations to a group of remotely located primary health centres (PHC) or community health centres (CHCs) which, as the "spokes", would be digitally connected to a designated college. PHCs and CHCs can be equipped with telehealth and teledentistry infrastructure to widen the reach of speciality care, facilitate education, training and provide better services to the society. With the success of the pilot implementation of CollabDDS, it is planned by the Ministry of Health and Family Welfare, Government of India to roll it out to all PHCs. CollabDDS Online Radiological Services includes 77 registered hospitals, 94 registered cases and 79 registered doctors. ${ }^{34,35}$

The pilot programme may be reviewed on an ongoing basis. Creation of a National Teledentistry Mission may be considered by the relevant statutory stakeholders, articulating amongst others, an appropriate institutional framework, norms and guidelines as well as an implementation plan to take this decentralised Hub-and-Spoke model nationwide.

\section{Conclusion}

Dentistry, in a synergistic combination with telecommunications technology and the Internet, has yielded a relatively new and exciting field that has endless potential. The use of teledentistry as a means to improve access to oral health services in areas with inadequate availability of general and speciality dental care is emerging as a practical solution in emergency aid, initial consultation and expert opinion. Currently, teledentistry has not yet become an integral part of mainstream oral healthcare system due to lack of administrative vision and financial constraints. There is also a need for enhanced public awareness of the advantages of telehealth for consultations, education and post-operative follow-ups. In the near future, with more successful applications like CollabDDS; teledentistry will be just another way to access an oral healthcare system. In spite of some unresolved issues, the potential of teledentistry is tremendous in the future in India.

\section{Corresponding author: \\ OP Kharbanda \\ Centre for Dental Education and Research \\ All India Institute of Medical Sciences \\ New Delhi \\ India \\ email:opk15@hotmail.com}

Conflict of interest. The authors declare no conflicts of interest.

Acknowledgement. The authors would like to acknowledge the CollabDDS team expert from the NIC New Delhi, Department of Radiodiagnosis, AIIMS, New Delhi, IIT-B, Mumbai, CSIO-CSIR, Chandigarh for their contribution in developing, implementation and improvision of CollabDDS System and providing data. The authors also to acknowledge State Nodal Officer of Maharashtra for the National Oral 
Health Program for providing data regarding telemedicine network in the state.

\section{References}

1. Census Report 2011. Available at: http://www.censusindia.gov.in accessed 4 November 2018.

2. National Health Programs in India. Available at: https://www.nhp.gov.in/healthprogramme/nationalhealth-programmes accessed 8 November 2018.

3. Life Expectancy in India; World Life Expectancy WHO Data 2018. Available at: https://www.worldlifeexpectancy.com/india-lifeexpectancy accessed 24 November 2018.

4. Shah N, Pandey R, Duggal R, Mathur U, Kumar R. Oral health survey in India: A report of multicentric study, WHO - Oral health survey 2004. Geneva, Switzerland: World Health Organization; 2005.

5. Lal S, Paul D. National oral health care programme (NOHCP) Implementation strategies. Indian J Community Med 2004;29(1):3-10. Available at: http://www.ijcm.org.in/temp/IndianJCommunityMed2 913-4459406_122314.pdf accessed 24 November 2018.

6. Operational Guideline National oral Health Programme. Ministry of Health and Family welfare. Govt of India 2012-2017. Available at: https://mohfw.gov.in/sites/default/files/513185637514 52762792_0.pdf accessed 8 November 2018.

7. Ayushman Bharat Scheme. Available at: http://ayushmanbharat.co.in/about-ayushman-bharatyojana accessed 3 April 2019.

8. Niranjan V. An overview of oral health plan of India: evaluating current status of oral healthcare and advocacy for national oral health policy. J Adv Oral Res 2015;6(3):24-29. DOI: 10.1177/2229411220150304

9. Vundavalli S. Dental manpower planning in India: Current scenario and future projections for the year 2020. Int Dent J 2014;64(1):62-67. DOI: 10.1111/idj.12063

10. Ready KV. Using teledentistry for providing the specialist access to rural Indians. Indian J Dent Res 2011;22(2):189. DOI:10.4103/0970-9290.84275

11. National Knowledge Network, India. Available at: http://nkn.gov.in/ accessed 8 November 2018.

12. Sood SP, Bhatia JS. Development of telemedicine technology in India: "Sanjeevani"- An integrated telemedicine application. J Postgrad Med 2005;51:308-11. Available at: http://www.jpgmonline.com/text.asp?2005/51/4/308/1 $\underline{9245}$ accessed 30 April 2019.
13. National Health Portal of India. 2016. Available at: https://www.nhp.gov.in/telemedicine _pg accessed 8 November 2018.

14. Department of Space- Indian Space Research Organization. Available at:

https://www.isro.gov.in/applications/telemedicine accessed 8 November 2018.

15. Sanjay Gandhi Post Graduate Institute of Medical Sciences (SGPGIMS) Telemedicine Program. 2012. Available at: http://www.sgpgitelemedicine.org/presentations.html accessed 8 November 2018.

16. Pan African e-Network Project (PAENP). 2013. Available at: https://www.mea.gov.in/Portal/ForeignRelation/Pan_A frican_e docx for xp.pdf accessed 20 November 2018.

17. Bari S, Arora P, Gupta AK, Singh M, Aggarwal AK. Tele-evidence: A videoconferencing tool as a viable alternative to physical appearance of doctors for the judicial summons. J Postgrad Med 2018;64(4):206211. DOI: $10.4103 /$ jpgm.JPGM_243_17

18. Maharashtra State Telemedicine Network. Available at: https://www.nrhm.maharashtra.gov.in/telemed.htm accessed 20 November 2018.

19. Telemedicine for rural mass- Current Initiatives and Future Scope. Available at: http://nmcn.in/nrc/ppt/Telemedicine\%20for\%20Rural $\%$ 20Masses \%20current\%20initiative\%20and\%20futur e\%20scope\%20Oditelecon.pdf accessed 20 November 2018.

20. Sudhamony S, Nandakumar K, BinuPJ, Niwas SI. Telemedicine and tele-health services for cancer-care delivery in India. IET Commun 2008;2(2):231-236. DOI:10.1049/iet-com:20060701

21. Telemedicine Society of India. 2018. Available at: http://www.tsi.org.in/ accessed 3 April 2019.

22. Project ECHO-India. 2018. Available at: https://www.echoindia.in/ accessed 8 November 2018.

23. Collaborative Digital Diagnosis System (CollabDDS). Available at: https://www.collabdds.gov.in accessed 8 November 2018.

24. eDantSeva. Available at: http://14.143.90.243/edent/ accessed 12 November 2018.

25. Legal aspects of Telemedicine. 2005. Available at: http://medind.nic.in/jbc/t05/i1/jbct05i1p3.pdf accessed 24 November 2018.

26. Golder DT, Brennan KA. Practicing dentistry in the age of telemedicine. J Am Dent Assoc 2000;131(6):734-744. DOI: 10.14219/jada.archive.2000.0272

27. Mathur P, Srivastava S, Lalchandani A, Mehta JL. Evolving role of telemedicine in health care delivery in India. Prim Health Care 2017;7(260):2167-1079. DOI: $\underline{10.4172 / 2167-1079.1000260}$ 
28. Department of Telecommunications. The Information Technology Act 2000. Available at:

https://www.meity.gov.in/content/informationtechnology-act-2000 accessed 20 November 2018.

29. Dasgupta A, Deb S. Telemedicine: A new horizon in public health in India. Indian J Community Med 2008;33(1):3-8. DOI: 10.4103/0970-0218.39234

30. Shirolkar R, RuparellaKP, More C, Ruparella P. Teledentistry: An art and science of healing. J Indian Acad Oral Med Radiol 2011;23(2):108-111.

DOI: 10.5005/jp-journals-10011-1106

31. Chandra G, Rao J, Singh K, Gupta K. Teledentistry in India: Time to deliver. J Educ Ethics Dent 2012;2:6164. Available at: http://www.jeed.in/text.asp?2012/2/2/61/121258 accessed 6 December 2018.

32. Nagarajappa R, Aapaliya P, Asawa K, et al. Teledentistry: Knowledge and attitudes among dentists in Udaipur, India. Oral Health Dent Manag 2013;12(3):138-144. DOI: $10.4172 / 2247$ 2452.1000502

33. Literacy in India. Available at: https://en.wikipedia.org/wiki/Literacy_in_India accessed 6 December 2018.

34. CollabDDS online radiological services. Available at: https://cors.gov.in/\%20 accessed 28 November 2018.

35. Mathews MA, Kathavate RN, Tewari S, Pawashe K. Teledentistry: A new frontier. Int J Oral Care Res 2015;3(4):52-57. Available at: http://www.ijocrweb.com/pdf/2015/OctoberDecember/9264_Review\%20Paper.pdf accessed 3 April 2019. 